\title{
Relação (incompleta) dos correspondentes de Adolpho Lutz
}

Abderhalden, Emil

Absolon, D. K.

Academia Brasileira de Sciencias

Academia Nacional de Medicina

Ackert, J. E.

Acott, Hugh

Adams, C. F.

Adams, Franklin

Affonso, Pedro barão de

Alexander, Charles P.

Alston, A. H. G.

Alves, Benedicto Ferreira

Amaral, Afrânio Peixoto Brasford do

Amaral, Ignacio Manoel Azevedo do

Andrade, Laerte Manhães de

Antunes, Waldemar

Arachavaleta, J.

Aragão, Henrique Beaurepaire Rohan

Araújo, Heráclides Cezar de Souza

Araujo, José Luiz de Barros

Araújo, Nestor

Armbruster, Humberto

Arp, Julius

Ashford, Bailey Kelly

Assis, Theodorico de

Astigas, Paulo de Toledo

Austen, E. E.

Azevedo, Thales

Azim, M. Abdul

Backmann

Bailhache, Preston H.

Baker, C. F.

Baliña, Pedro L.

Bambarén, Carlos A.

Bancroft, Joseph

Banks, Charles S.

Barbiellini, Amadeu A.

Barbosa, Emílio de Castilho

Barbosa, Plácido

Barbour, Thomas

Barretto, José Pereira

Barros, W. Duarte de

Barth, Rudolf

Bassewitz, Ernst von

Baumann, F.

Bausenhardt
Baylis, H. A.

Bayma, Theodoro

Beach, Frank A.

Beebe, William

Behr, Anton

Beier, M.

Bello, Joaquim Ferreira

Benecke, W.

Benko, André

Benny, George P.

Bercovitz, Z.

Berlesi, Antonio

Bernard, R.

Bernstein, Joseph

Beurmann

Beurmann, Lucien de

Bezzi, Mario

Bhaduri, L. L.

Biblioteca Nacional

Bigalke, R.

Billet, A.

Billings, A. W. K.

Birkenmeier, Elmar

Blair, W. Frank

Blanchard, Raphaël

Blanco, Ramon Alfonzo

Bleyer, Jorge Clarke

Blüth, Alfred

Boesel, M. W.

Bogert, Charles M.

Borgmeier, P. Thomas

Boris

Botelho, Carlos

Bourroul, Celestino

Bowman, F. H.

Boyce, Robert

Boyd, M. F.

Bradley, J. Chester

Braestrup, F. W.

Bragg, Arthur N.

Brandes

Braun, Walter C.

Braunsburger, Leo

Bree, Peter J. H. van

Breen, John F.

Breitkopf \& Härtel 
Bresslau, Ernst

Brethes, Juan

Brito, Luiz Edmundo Rangel de Souza

Britto, Alfredo

Broden, A.

Brongersma, L. D.

Brownell, L. W.

Bruch, Frank Carl

Brunn, M. V.

Bueno, A. Dino

Buhrnheim, Paulo Friedrich

Bukuwky, Michael

Bülow, Carl Adolf

Burger, W. Leslie

Burgess, Perry

Burnet, Etienne

Burroughs, R. A.

Burt, Charles E.

Buschke

Bustamante, Fernando

Cabrera, Ricardo Pérez

Calcagno, R.

Cameron, A. E.

Campello, F.

Carini, Antonio

Carter, Henry F.

Carvalho, Octavio de

Cassino, Samuel

Castro, Germiniano de Lyra

Castro, Gustavo Mendes de Oliveira

Castro, Lyra

Cerqueira, Dionisio

Cerqueira, Gama

Cerqueira, J.

Chagas, Carlos

Chapot-Prévost, Eduardo

Chaves, Leocadio

Chen, T. G.

Chittenden, F. H.

Christopherson, J. B.

Chun, Carl

Ciurca, Joan

Cleave, H. J. van

Cochran, Doris M.

Cochrane, Robert G.

Coni, Emílio R.

Connor, M. E

Coquillett, D. W.
Cordeiro, Ergasto H.

Cordes, T.

Cort, W. W.

Costa, Eduardo Borges da

Costa, Sylvio Celso Gonçalves da

Cotrim, Eduardo

Couto, Miguel

Creaser, Edwin P.

Crowell, Bowman C.

Cruz, Oswaldo Gonçalves

Cruz, Venâncio

Cuéton, B. L.

Cumming, H. S.

Cunha, Raul Leitão da

Dale, João

Damazio, Leonidas B.

Daniels, W.

Dansereau, Pierre

Davis, Nelson C.

Deijskes, D. C.

Delfino, Victor

Denys, Major

Devenish, Syl

Dias, Ezequiel Caetano

Dice, L. R.

Dickerson, $\mathrm{T}$.

Dickson, Daphne

Dijke, M. J. v.

Digalke, B.

Diretoria do Museu Nacional

Divisão de Zoologia

Dolphus, Robert

Dreher, Otto

Dubois, Georges

Ducke, Adolpho J. W.

Dunbar, William Philipps

Dundee, Harold A.

Dunn, Emmet R.

Dunn, L. H.

Dunn, Merle

Dupuy

Dymond, J. R.

Eckstein

Edwall, Gustav

Edwards

Ehrhardt, Wilhelen

Ehrlich, Paul

Eiselt, Josef 
Eitel, Wilhelm

Ejsmont, Leopold

Elton, Charles

Emerson, Haven

Escomel, Edmundo

Fairchild, A. G. B.

Fajardo, Francisco

Faust, Edwin P.

Faust, Edwin S.

Fenwick, Charles G.

Ferenoz, José

Fernandes, Eurico

Ferris, R. S.

Ficker, Martin

Fidanza

Fiebrig, Karl

Figueiredo, Amazonas

Figueiredo, Bonifácio

Finch, Amanda

Finneran, L. C.

Fischer, Carlos Rudolph

Fischer, Gustav

Flint, W. P.

Floch, Hervé

Flores, Román Sabas

Foetterle, J. G.

Fonseca Filho, Olympio de Oliveira Ribeiro da

Fonseca, Flavio da

Fonseca, José Pinto da

Fontes, Antonio Cardoso

Forattini, Oswaldo Paulo

Forel, Auguste-Henri

Forster, Hans

Foster, Racine

Fraga, Clementino

Freiberg, M. A.

Freire, Oscar

Freise, Frederico W.

Freitas, J. F. Teixeira de

Frenz

Frison, Theodore $\mathrm{H}$.

Fróes, Heitor Praguer

Fuhrmann, Otto

Fujiama, Akira

Fülleborn, Friederich

Gaertner, Erich

Gaiger, Helen

Gallardo, José Maria
Garré

Gay, Hazel

Gelder, Richard G. Van

Giacomelli, Eugène

Gibson, Arthur

Giesler, Paul

Gijs, P. de

Gilmour

Gleason, H. A.

Gliesch, Rudolf

Globig, Hermann

Gloyd, Howard K.

Goebel, K.

Gofferjé, C. N.

Goin, Coleman J.

Gomes, A.

Gomes, Delir Corrêa

Gomes, Emílio Emiliano

Goñalons, G. P.

Gonçalves, Álvaro

Gordon, Robert E.

Gouveia, Hilário de

Graf, J. E.

Grassi, Giovanni Batista

Gregory, Margaret P.

Grobman, Arnold B.

Guedes, Pinto

Guerra, E. Salles

Günther, Hugo

Guravich, D.

Gurgel, Luiz do Nascimento

Gurney

Güthner, Karl

Hackett, L. W.

Hall, E. R.

Hammar, A. G.

Hardy Jr., Jerry D.

Harris, Benita S.

Hase, Albrecht

Haslinger e Pessek

Hassal, Albert

Hassinger, B.

Haupt, Octavio de Aguiar

Hayashi, Fumio

Hecht, Bessie Matalas

Hediger, $\mathrm{H}$.

Heini

Heinroth 
Heiser, Victor George

Hellmich, W.

Hempel, Adolph

Heney, Carlos C.

Henriquez, Antonio

Heringer, Ezechias Paulo

Heymann, Bruno

Heymont, R.

Hill, Howard A.

Hine, James S.

Hinman, E. Harold

Hochwolgeboren, Euer

Hoehne, F. C.

Hoffman, Frederick Ludwig

Hoffmann, Wilhelm $\mathrm{H}$.

Hoffstetter, Robert

Hohl, Andreas

Honigmann, Hans Leo

Hora, S. L.

Horn, Walter

Horta, Almada

Horta, Paulo Parreiras

Hottinger, Rob

Houba, Renée

Houssay, Bernardo A.

Howard, Leland Ossian

Howard, Richard A.

Huber, J. Ch.

Hudson, N. Paul

Hughes, R. Chester

Hull, Frank W.

Hume, Edgar Erskine

Hunter, W. D.

Huxley, Julian S.

Ibarra, Roberto Alamo

Igaravidez, Gutierrez

Ihering, Hermann von

Ihering, Rodolpho von

Immel, Bruno

Infante, Ramon Pineda

Iwata, Seishun

Jackson, Stedman W.

Jacobs, S.

Jaffé, Rudolph

Jameson, David L.

Janicki, C.

Jannot, M.

Janson, Harvey S.
Jean, Yves

Jensen, $H$.

Johannsen, O. A.

Johnstone, Herbert

Joseph, Gustav

Joyeux, Ch.

Juan e 'Roiz'

Junge, Carlos

Jungmann, Herbert A.

Jurado, Martin Doello

Jurberg, José

Kagan, Irving G.

Kapiolani, H. M. Queen

Kauntze, Willian Henry

Kellog, Peter Paul

Kern, Carlos R.

Kertész, K.

Kicherer, A. H.

Killip, E. P.

Klauber, Laurence M.

Klinke, Carlos

Knab, Frederick

Knoblauch, August

Kobayoshi, Harujiro

Kohda, Kohtaro

Kolle, W.

Komiya, Yoshitaka

Kömpel

Koppel, C. Vans de

Kotlán, A.

Kraepelin, K.

Krause, Franz

Krauss, Rudolff

Krichbaum, Jorge

Kröber, O.

Krukoff, B. A.

Kudo, R.

Kumm, Henry W.

Küntzell

La Rue, George R.

La Teya, Carlos Napoleão

Laboratório Paulista de Biologia

Lacerda, J. B. de

Ladeira, Onofre

Lage, Helly Arruda

Lamartine, J.

Lamartine, Oswaldo

Lamotte, Maxime 
Lane, Lauriston Job

Lange, Rudolph B.

Lankester

Lark, P.

Lassen, Verner

Latham, V. A.

Laven, $\mathrm{H}$.

Laveran, Alphonso

Lawrence, W. H.

Leão, Aristides Azevedo Pacheco

Leavitt, Benjamin B.

Leboullanger, L.

Ledoux, Paul

Léger, L.

Lehman, H. E.

Lehrman, Daniel S.

Leiper, Robert Thompson

Leite, Juvenal

Leloas, $\mathrm{H}$.

Leme, Alberto Betim Paes

Leme, João B. da Silveira

Lent, Hermann

Lenz, Friedrich

Léssa, Luis

Lestage, J. A.

Lester, J. W.

Leuenberger, S. Gottfried

Leurat

Libero, Kandio

Liebermann, José

Lima, Alvarez

Lima, José Jerônimo de Azevedo

Lima, Campos

Lima, Carvalho

Lima, Henrique da Rocha

Lima, Luiz Nascimento

Lima, Rubens Rodrigues

Lindner, Erwin

Linhares, Augusto

L'Institut Zoologique de l'Université de Varsovie

Lintow, Edwin

Lisbôa Junior, José M.

List, C.

Livezey, Robert L.

Lobo, Bruno Álvares da Silva

Lochhead, John H.

Lohmann, Carlos Ernesto Julius
Lohner, F. A.

London School of Hygiene and Tropical

Medicine

Looss, Arthur

Lopes, Hugo de Souza

Loveridge, Arthur

Lowen, L.

Luce, Helena Lutz

Luce, Godofredo Wilken

Luederwaldt, $\mathrm{H}$.

Lühe

Luther, John

Lutz, Bertha

Lynn, W.

MacFarlane, Andrew

Machado, Virgílio

Maciel Filho, Abel Pinheiro

Maciel, Jesuino

Mac Donagh, Emiliano G.

Mackie, Thomas T.

Madeira, Daniel

Maeder, P.

Magalhães, José Augusto de

Magalhães, Octavio de

Magalhães, Pedro Severiano de

Maia, Luiz Carlos

Malloch, J. R.

Malta, Francisco de Toledo

Mann, Lucile

Mann, W. M.

Manson, Patrick

Marherr, Erich

Maramoez, P. Rimenez

Marinho, João

Martin, T. S.

Martinez, Ezequiel A.

Martinez, Gonzalez

Martins, Alceu Osias

Martins, Almicar Viana

Martins, Carlos

Martins, Oliveira

Massart, J.

Matta, Alfredo Augusto da

Maurano, Flavio

Mayr, Ernst

Mazza, Salvador

McBride, K.

McClung, C. E. 
Mehra, H. R.

Melo, Oswaldo de

Mendes, Dario

Mendheim, Hans

Mendonça, Arthur

Meneses, Pedro Telles Barreto de

Menzies, J. J.

Mercier, Maria de Lourdes

Merége, João Rodrigues de

Merrui, P.

Mertens, Robert

Metcalf, C. L.

Metcalf, Maynard M.

Metchnikoff, Élie

Meyer, Carlos

Meyers, George S.

Michaelsen, W.

Mickel, Clarence E.

Migone, Luis E.

Miller, Harry M.

Minssen, Guilherme

Miranda, F. G. Valle

Miranda, Severiano de

Miyajima, M.

Moldenke, Harold N.

Mole, R. R.

Momura, Keyri

Moniz, Sylvio

Monte, Oscar

Monteiro, Félix

Monteiro, W.

Montes

Montgomery, D. U.

Monticelli, Francisco Saveiro

Moreau, Julio E.

Moreira, Carlos

Morrison-Scott, T. C. S.

Moses, Arthur Alexandre

Motta, Catarina da Silva

Moulton, Dudley

Moura, Carlos

Moura, Nitia de

Muhlens

Muir, Ernest

Müller, Lorenz

Murce, Rocha

Naderer, Karl

Nakarai, S.
Neave, Sheffield A.

Necker, Walter L.

Neiva, Arthur

Netting, M. Graham

Neves, José Aroeira

Newstaed, Robert

Niekau, Bruno

Noble, G. Kingsley

Nocht, Bernhard

Nutalt, N. F.

Nuttall, George H. F.

Oberndorfer, Eugen Albrecht

Oergel

Ofuhman

Oliveira, Fabrício Caldas de

Oliveira, Jayme de

Oliveira, Lejeune Henrique Pacheco de

Oliveira, Numa Pires de

Oliveira, Olinto de

Oliveira, Sebastião José de

Ortiz, Jonas

Orton, William A.

Pacheco, Genesio

Pakf, Helene

Palombi, Arturo

Parker, H. L.

Parker, H. W.

Parona, Corrado

Passareli, A.

Patiño, José Luis Rios

Patton, W. S.

Regendanz, Paul

Paulo, Vicente de

Paulsen, Johannes

Pearce, Richard M.

Peckolt, Gustavo

Peña

Pereira, Octaviano de Loyola

Perroncito, Edoardo

Peschkowsky, Georg

Pestana, Bruno Rangel

Pestana, Nestor Rangel

Pestana, Sebastião

Pestre, R.

Peters, James A.

Peutz, Kathleen

Pfeeffer, Georg

Pfeiffer, Joseph 
Pfeiffer, L.

Pflaumer, Karl

Picaro, F.

Pickel, Bento

Pierantoni

Pigulewsky, Serguei Wladimirowich

Pinto, Cesar

Pinto, Getulino Vieira

Pinto, Gil Sobral

Pinto, Oliverio Mario de Oliveira

Pirajá da Silva, Manuel Augusto

Pires, Heliodoro

Pittier, Henry

Piza, José de Toledo

Plaut, H. C.

Poll, M.

Pompeu, João B.

Poncy, A.

Pontes de Miranda, Francisco Cavalcanti

Pope, Clifford $\mathrm{H}$.

Portugal, Olympio

Pound, G. J.

Prado, J. Leal

Prévost, Gustave

Price, Emment W.

Puente, J. J.

Puttermann, $\mathrm{H}$.

Rand, Frederich V.

Raney, Edward C.

Ranger, Stanley G.

Ranson, B. H.

Raposo, Hélio

Rausam, M.

Ravenel, W. de C.

Regendanz, Paul

Rêgo, Amilcar Arandas

Rehl, João

Rehn, James A. G.

Reis, Andrade

Rensch, Bernhard

Rhind, C. B.

Rhoades, Rendel

Ribas, Emílio Emiliano

Ribeiro, Alípio de Miranda

Ricardo, Gertrude

Rice, Howard J.

Riesenfeld, Ludwig

Riley, A.
Risquez, Jesus Rafael

Rivero, Juan A.

Robinson, Helene M.

Rocha, Francisco Dias da

Rocha, Ismael da

Rocholl, Carlos

Rodhain, J.

Rodrigues, Henrique de Oliveira

Rodrigues, Manoel

Rodrigues, Nina

Rodríguez, Juvenal Valerio

Roedl, Johannes

Roenve, Hans

Rohwer, S. A.

Rolfs, P. H.

Rolle, Hermann

Rollo, Ruth L.

Römer, Fritz

Ronna, Antonio

Rose, Wickliffe

Rosen, M. J.

Rosenstirn, Jul.

Ross, Herbert H.

Ross, M. C.

Rothschild, Miriam

Rothschuh, E.

Roux, Jean

Rowan, W.

Ruata, Guido

Rudolph, Max

Ruge, J.

Rühl

Ruschi, Augusto

Ruszkowski, J. S.

Sãbgra, Alfredo

Sachs, William F.

Sachtleben, Hans

Sallmann, Carl

Salmon, E.

Sampaio, José de Campos

Samuelsson, G.

Sandig, Paul

Santamaria \& Presch — Livraria Tupy

Santos, Manuel Camilo dos

Santos, Paulino F.

Saporiti, Enrique J.

Sapucaia, Alfredo Gomes

Savage, Jay M. 
Sawaya, Paulo

Sawyer, Willburn A.

Schachner, August

Schaeffer, Alfredo

Schnee, Paul

Schern, Kurt

Schimdt, Karl P.

Schirch, Paul

Schlünzen, Martha

Schlünzen, Praeses

Schmalz, João Paulo

Schmalz, Marie

Schmalz, Otto

Schmidt, Fritz

Schmidt, Karl P.

Scholl, Otto

Schomps, Fleo U.

Schönfeldt

Schouten, Guillermo B.

Schreiber, Giorgio

Schuster, Ortrud

Schutte, Julio F.

Segan, Mildred

Seidel, Max

Seme, Carmelia B. da Silveira

Seng, Walther

Sergent, E.

Serié, Pedro

Shreve, Benjamin

Sick, Helmut

Sidwell, Gloria

Silva Araújo, Oscar da

Silva, Flaviano

Silvafranjos, Paulo

Silva, Manoel Augusto Pirajá da

Silva, Maurilio Pinto da

Silveira, Álvaro Astolpho da

Silveira, Guilhermino M.

Simões e Silva Júnior

Simond

Simplício, João

Smith, Albert G.

Smith, Hobart M.

Smith, Lyman B.

Smith, Philip W.

Smith, Vera Irwin

Sociedade Brasileira de Dermatologia

Sociedade Brasileira de Sciencias
Sodré, Antônio Augusto de Azevedo

Sollner

Soparkar, M. B.

Soper, Fred L.

Sophia, A.

Sousa, Geraldo H. de Paula

Southwick, Margareth D.

Souza Seabra \& Cia. Ltda.

Spegazzini, Carlos

Speiser, Paul

Splendore, A.

Stahel, Gerald

Starfford, W.

Staudacher, A. F.

Stefanopulo

Stenberg, Hilgard O' Reilly

Sternberg, George Miller

Stickel, Lucille F.

Stickel, William H.

Stilles, Charles W.

Strain, Willian London

Strauss, Gunda

Striker, Helena V.

Strode, George K.

Stuart, L. C.

Sturmhöfel, R.

Swanson, Gustav

Szidat, Lothar

Tanabe, B.

Taunay, Affonso d'Escragnolle

Taylor, Edward H.

Teisen, Aase

Teixeira, J. de Mello

Teixeira, Nothel

Tejera, Enrique

Terni, Camillo

Terra, Fernando

Test, Frederick H.

Thienemann, August

Thomas, Hyell J.

Thomas, M. A.

Thomas, Ruth S.

Thompson, J. Ashburton

Tibiriça, Alice de Toledo Ribas

Todas, S.

Toledo, Bonilha de

Torres, Heloisa Alberto

Torres, Octavio 
JAIME L. BENCHIMOL, MAGALI R. SÁ, JOHANN BECKER et alii

Tovar, Manuel Nuñez

Townsend, C. H. T.

Trapidas, Harold

Travassos Filho, Lauro Pereira

Trivass, Montajue A.

Tuker, R.

Twight, Clodomiro Picado

Ufelander, A. L.

Uffenheimer, Albert

Unna, Paul Gerson

Valerio, Manuel

Vanzolini, P. E.

Velasco, Mario

Vellard, Jehan Albert

Velloso, Henrique

Vermuth, Heinz

Vicente, Joaquim Julio

Vickers, H. M.

Vieira, Francisco Borges

Viets, Karl

Villeneuve, J.

Villiers, C. G. S. de

Vincent, George E.

Vitzthum, H. Graf

Vogesland, E. G.

Vogl, Cornelius

Vogt, Karl

Wade, H. W.

Wagstaff, W. G.

Walker, Ernest P.

Walther, Johannes

Walther-Gimbel, Leonhard Alfred

Wancolle, Alexandre

Ward, Henry Baldwin

Watson, Malcom

Weber, F.
Weld, William H.

Weltner, $\mathrm{H}$.

Weltner, W.

Werler, John E.

Werner, Elisabeth

Wernicke, Roberto

Wesenberg-Lund, C.

Wetmore, A.

Wfeukeiman

Whatmough, G.

White, Norman

Wickborn

Wiggermann, P. Gebardo

Wilder, Francis

Wildt, B.

Wille, Johannes

Williams, Francis

Wilson, D. B.

Wilson, Robert W.

Wisniewski, L. W.

Wolffhügel, Kurt

Wolterstooff, W.

Wood, John Thornton

Wytsman, P.

Xima, Emile

Yakogawa, S.

Yamagushi, Saytô

Yoshida, Sadao

Young, Ernest

Zankester, E. Ray

Zehntner, Leo

Ziemann, $\mathrm{H}$.

Zikán, José Francisco

Zinku, Karl

Zschokke-Dietschy, Fritz 